\title{
Teoría decolonial y estudios sobre hábitat: construcción de un soporte epistemológico desde el enfoque de comunicación e interculturalidad
}

Decolonial theory and studies about habitat: construction of an epistemological support from the communication and interculturality approach

Facundo David Francisco González facundoinenco@gmail.com https://orcid.org/0000-0002-1603-4235

Instituto de Investigaciones en Energía No Convencional; Facultad de Ciencias Exactas; Universidad Nacional de Salta/ Consejo Nacional de Investigaciones Científicas y Técnicas

(Argentina)

Nuria Macarena Rodríguez nuria.macarena.rodriguez@gmail.com https://orcid.org/0000-0003-3692-294X

Instituto de Investigaciones en Ciencias Sociales y Humanidades; Universidad Nacional de Salta/ Consejo Nacional de Investigaciones Científicas y Técnicas (Argentina) 


\section{Resumen}

El presente trabajo tiene como objetivo reflexionar acerca del potencial teórico-epistemológico del enfoque comunicación/interculturalidad para el abordaje de los procesos de producción social de hábitat. La propuesta emerge a partir de un proyecto de extensión/investigación para la transferencia de tecnologías a base de biomasa en la cocina de la escuela de una comunidad indígena wichi del chaco salteño. La experiencia en el campo provocó una ruptura con el enfoque transferencista y derivó en un replanteo de los supuestos epistemológicos que sustentaban la intervención. Así, entendemos a la comunicación como un escenario de relaciones de cultura que giran en torno a la habitabilidad y que tienen diferentes motivaciones: resolver necesidades básicas como el alimento, el trabajo, el acceso al agua potable y a la energía, ocupación del tiempo libre, entre otros. La particularidad del caso en estudio, que incluye procesos de interacciones y negociaciones para la producción de hábitat, demandó pensar estrategias que permitieran poder reconocernos en la diversidad y comunicarnos sin invisibilizarla, ignorarla o desconocerla. La opción epistemológica asumida para tal fin se apoyó en la teoría decolonial desde la cual no podemos pensar los procesos de producción de sentido en hábitat por fuera de la perspectiva intercultural.

Palabras clave: Hábitat; comunicación; decolonialidad; interculturalidad.

\section{Abstract}

The present work has like objective to reflect about of the theoretical-epistemological potential of the approach communication/interculturality for the focusing of the processes of social production of hábitat. The proposal emerges from an extension/research project for the transfer of technologies based on biomass in the kitchen of the school of an indigenous community wichi of Chaco salteño. The experience in the field caused a break with the transference approach and led to a restatement of the epistemological assumptions that supported the intervention. Thus, we understand communication as a scenario of cultural relations that revolve around habitability and that have different motivations: to solve basic needs such as food, work, access to drinking water and energy, occupation of free time, among others. The particularity of the case under study, which includes processes of interactions and negotiations for the production of habitat, demanded to think strategies that would allow us to recognize ourselves in diversity and communicate without making it invisible, not know it or ignore it. The epistemological option assumed for this purpose was based on the decolonial theory from which we cannot think about the processes of sense production in habitat outside the intercultural perspective.

Key words: Habitat; communication; decoloniality; interculturality. 
El objetivo central de este artículo consiste en analizar el potencial teórico epistemológico de la relación comunicación/interculturalidad, en el marco paradigmático de la teoría decolonial, para abordar procesos complejos de producción social de hábitat.

La experiencia soporte es un proyecto de investigación/extensión (1) en el Chaco salteño denominado La Misión Rivadavia: diagnóstico e intervención socio técnica para el mejoramiento del hábitat de comunidades originarias wichí en el Chaco salteño. Allí se desarrolló una tarea de implementación de tecnologías a base de biomasa en la cocina de una Escuela rural bilingüe de la comunidad indígena wichí "El Cocal", ubicada a 30 kilómetros de Rivadavia Banda Sur, donde viven 20 familias.

El proyecto se planteó, desde la primera etapa, con la intención de realizarlo con un enfoque participativo. No obstante, las diferencias culturales se presentaron como un elemento a tener en cuenta que no se solucionó "sólo escuchándolos". Así pues, emergió la necesidad de construir un dispositivo de comunicación que permitiera la interacción entre personas culturalmente distintas, pero capaces de reconocerse mutuamente y comunicarse (2). El proyecto, que técnicamente solo consistía en transferir (3) a la Escuela de El Cocal, cocinas eficientes que aprovechan la chamiza (hojas y ramas caídas de los bosques aledaños a la comunidad) como combustible, fue tomando dimensiones inesperadas ya que el proceso de apropiación e incorporación de los equipos a la rutina de los miembros de la escuela excedía la simple enseñanza sobre los modos de utilización del equipo tecnológico.

Ante esta situación y obligados por los emergentes de la primera etapa del trabajo de campo, asumimos la necesidad de incluir elementos de la perspectiva Intercultural. La intencionalidad primera, fue la de incorporar una herramienta que aportara una perspectiva crítica que optimizara el proceso de interrelación entre actores culturalmente distintos, y en cómo estos actores se constituyen, consolidan e interactúan.

\section{Estudiar los procesos de producción de sentidos en la construcción de hábitat ¿Desde la comunicación?}

\section{La discusión del estatuto epistemológico de la comunicación como ciencia}

La comunicación, como campo de conocimiento, sin una tradición disciplinar enraizada y delimitada -objeto de crítica de muchos autores- es más bien percibida como una ventaja en el marco del enfoque de la postdisciplinariedad. Al respecto, Raúl Fuentes Navarro señala que la comunicación "apuesta por la superación de las fronteras existentes entre las disciplinas, por la integración metodológica (...) por el abandono del afán de disciplinar su estudio; esto es, por la negación de una posibilidad de una comunicología" (1998: 16). Esta posición se contrapone con la opinión de otros referentes del campo, como Jesús Galindo, quien plantea desarrollar una "Comunicología posible mediante la articulación, en una matriz, de las vertientes analíticas de la difusión masiva, la terapia interpersonal y los estudios culturales "(Galindo, 2003: 10). Sobre el estatuto epistemológico de la comunicación, León Duarte (2006) ha señalado que la perspectiva sociocultural se constituye en la visión predominante de la investigación académica, dentro de un contexto de prácticas de investigación orientadas a las ciencias sociales, y con fuerte demanda de definición precisa del estatuto disciplinario. De modo tal que el estatuto disciplinar de las ciencias de la comunicación evolucionó hacia la superación de los límites entre las especialidades de los campos de conocimiento. Así pues, entendemos a la comunicación como un campo transhistórico, sociocultural y complejo, multidimensional,

Question, Vol. 1, N. ${ }^{\circ} 63$, julio-septiembre 2019. ISSN 1669-6581

Instituto de Investigaciones en Comunicación | Facultad de Periodismo y Comunicación Social | Universidad Nacional de La Plata La Plata | Buenos Aires | Argentina 
centrado en la subjetividad y en los procesos de producción de sentido. En consecuencia, el pensamiento pos y transdisciplinar representan una perspectiva relativamente reciente, originada en el proceso histórico de la globalización (Moyano, 2016: 24).

Torrico Villanueva señala que el objeto de estudio de la comunicación:

\begin{abstract}
(...) es el proceso, circulación mediada, intercambio desigual, intelección, y uso de significaciones y sentidos culturalmente situados, que es algo de naturaleza socialmente estructural e inseparable -para fines teóricos e investigativos- de las otras dimensiones analíticas de la vida social que se estudian (2004: 31).
\end{abstract}

Este autor advierte que la definición antes señalada responde al objeto teórico de la comunicación. En cambio, serán objetos concretos los procesos comunicacionales reales sometidos a observación y estudio.

La utilización de este concepto pretende guardar coherencia con una concepción de la comunicación que se apoya, sobre todo, en las relaciones y en las interacciones entre los sujetos que son actores de esta (Uranga, 2017). Concretamente, se está refiriendo a las prácticas de comunicación que se verifican y pueden ser reconocidas en el contexto de las prácticas sociales. Es decir, a situaciones de comunicación protagonizadas por actores, individuales o colectivos, en permanente dinamismo y situados en proyectos, en organizaciones, en instituciones. Los procesos comunicacionales hacen referencia a los actores, a espacios de necesidades y demandas comunicacionales vinculadas con las prácticas que se verifican en la sociedad (Uranga, 2017).

La multidimensionalidad, característica del objeto de estudio de la comunicación, tiene gran consenso en el campo. Esto se debe a que su cualidad estructural atraviesa prácticamente todos los ámbitos de la existencia y la realidad social es transversal a ellos.

En este contexto, es posible configurar como tema pertinente los estudios sobre hábitat, como dimensión macro, constituyente de la vida humana. La noción de hábitat refiere al ser y estar en la tierra:

Esto va más allá de usar, ocupar, radicarse en o protegerse debajo de un artefacto tecnológico, puesto que el proceso dinámico de hábitat resulta de la confluencia de planos diferentes, analíticamente distinguibles entre lo natural, social, económico, cultural, político, emocional, físico-espacial, tecnológico, entre otros (Vanoli, Martinez y Cejas, 2018: 4).

Así pues, entendemos a la comunicación como un escenario de relaciones de cultura (Ceballos Guerra, 2015). Esas relaciones que giran en torno a la habitabilidad y que tienen diferentes motivaciones: resolver necesidades básicas como el alimento, el trabajo, el acceso al agua potable y a la energía, ocupación del tiempo libre, entre otros. Juan Carlos Ceballos Guerra agrega a las motivaciones mencionadas, las necesidades del ser humano en su convivencia social como el afecto, el diálogo, la participación.

Evidentemente, hábitat está ligado a la noción de construir, de ser, de estar en el territorio. Sobre esto, Noelia Cejas (2014) asegura que habitar implica reconocer en cada uno de los agentes partícipes del proceso de hábitat "su capacidad intrínseca de producir significados en todos los órdenes que pudieran emerger del proceso" (p. 98).

La construcción del objeto. ¿Por qué es posible investigar procesos de producción de hábitat desde la comunicación?

La sociedad genera, de manera continua, redes de comunicación (interacciones significativas, intercambios) que se materializan en los espacios físicos y en escenarios culturales. Y en la forma de habitar, se dan prácticas y procesos de comunicación que están presentes en la

Question, Vol. 1, N. ${ }^{\circ}$ 63, julio-septiembre 2019. ISSN 1669-6581

Instituto de Investigaciones en Comunicación | Facultad de Periodismo y Comunicación Social | Universidad Nacional de La Plata La Plata | Buenos Aires | Argentina

Página 4 de 10 
construcción colectiva de memorias, lenguajes, tecnologías, y símbolos (Ceballos Guerra, 2015). Esos espacios de intercambios y relaciones expresan significados que las personas construyen en las prácticas de habitabilidad.

Para comprender el hábitat, adherimos al concepto de Producción Social de Hábitat (PSH), que amplía y actualiza el concepto de progresividad, que fue desarrollado por miembros de distintas organizaciones del Hábitat Internacional Coalition (HIC) interesados en la defensa del derecho al hábitat, definiéndolo como:

\begin{abstract}
Todos aquellos procesos generadores de espacios habitables, componentes urbanos y rurales, que se realizan bajo el control de autoproductores y otros agentes sociales que operan sin fines lucrativos. Parte de la conceptualización de la vivienda y el hábitat como producto social y cultural y no como mercancía, como acto de habitar y no como mero objeto de intercambio (Enet, 2008).
\end{abstract}

El proceso de PDH implica -señala Mariana Enet- una nueva forma de gestión y de acción que no responde al modelo dominante, sino a una cultura de la solidaridad y de la complementariedad con otros actores, con implicancias políticas, económicas y sociales de transformación de las relaciones de poder. A su vez, necesita de la participación activa de los pobladores y estar apoyado por asistencia técnica interdisciplinaria.

Para comprender la complejidad de los procesos de hábitat, abordados desde el enfoque de la $\mathrm{PSH}$, consideramos esencial analizar los procesos comunicacionales, ya que permiten una aproximación a la configuración del hábitat como problema del Estado y de otros actores. Las políticas públicas, como conjunto de objetivos, decisiones y acciones que lleva a cabo un gobierno para solucionar los problemas que en un momento determinado los ciudadanos y el propio Estado consideran prioritarios (Tamayo Sánchez, 1997), son instrumentos que ayudan a entender cuándo un problema tiene reconocimiento estatal. Así, las políticas públicas que el Estado implementa para el mejoramiento del hábitat permitirán vislumbrar su enfoque en la producción de hábitat. En el caso de la experiencia que mencionamos como objeto de este trabajo, señalamos que pertenece al rubro de políticas públicas orientadas al mejoramiento del hábitat.

La experiencia del proyecto de extensión, nacida del encuentro entre técnicos (investigadores, estudiantes y científicos del Instituto de Investigaciones en Energía No Convencional) y comunidades originarias del chaco salteño, presume un intercambio de saberes pretendidamente- horizontal, desde el momento de ejecución de los proyectos, hasta la convicción propia de los participantes de la necesidad de trabajar conjuntamente en la mejora integral de su hábitat.

Entendemos que la clave comunicacional es un punto privilegiado a la hora de pensar los procesos participativos de producción de conocimiento (Vanoli et al., 2018: 4) y la gestión de proyectos que intervengan el hábitat de una comunidad.

\title{
Opción paradigmática: comunicación decolonial
}

El paradigma teórico -y político - como marco articulado de conceptos "desde" o "a través" del cual se puede mirar una zona de la realidad social para comprenderla- que mejor se adecua a los propósitos del proyecto, es el decolonial, apoyado en el Programa de Investigación Modernidad/Colonialidad (Escobar, 2003) o también llamado Proyecto latino/latinoamericano modernidad/colonialidad (Castro-Gómez y Grosfoguel, 2007).

En tal sentido, entendemos como paradigma epistemológico -o espacio de enunciación - al "sistema básico de creencia o visión de mundo que guía al investigador, ya no sólo al elegir métodos, sino en formas que son ontológica y epistemológicamente fundamentales" (Guba y Lincoln, 2002: 58). Claramente, este posicionamiento se aleja de la noción eurocentrista

Question, Vol. 1, N. ${ }^{\circ}$ 63, julio-septiembre 2019. ISSN 1669-6581

Instituto de Investigaciones en Comunicación | Facultad de Periodismo y Comunicación Social | Universidad Nacional de La Plata La Plata | Buenos Aires | Argentina

Página 5 de 10 
kuhniana puesto que, además de pensar los patrones de investigación como concepción teórica, se hace cargo de una posición ideológico-política. Más que un paradigma de transición, es un paradigma de disrupción (Mignolo, 2003).

El Programa de Investigación Modernidad/Colonialidad cuestiona no sólo los conceptos utilizados para dar cuenta de los procesos sociales de la región, sino además los presupuestos paradigmáticos que los sustentan, sumado a que "se orientan a la conformación de un paradigma otro" (Mignolo, 2003). Ese paradigma otro, da lugar a una comunicación otra. Allí, el pensamiento decolonial emerge de las experiencias marcadas por la colonialidad y está formulado desde la exterioridad de la modernidad, por lo que se encuentra en condiciones de aportar una comprensión distinta, no eurocéntrica, de la dominación y la explotación: un modo otro de ver.

Sobre esto, Erick Torrico Villanueva sostiene que:

El programa se inspira particularmente en la historia de sojuzgamiento vivida por los pueblos indígenas y afro y somete a juicio la triple colonialidad que sustenta el régimen moderno del capital: la colonialidad del poder (Quijano, 2000), la colonialidad del ser (Nelson Maldonado Torres) y la colonialidad del saber (Lander, 2000) (Torrico Villanueva, 2010: 69).

En la dimensión epistémica, sostiene Torrico Villanueva, la decolonialidad desautoriza la racionalidad moderna que separa naturaleza de sociedad, sujeto de objeto o lo arcaico de lo moderno, concluyendo que: "Este pensamiento fronterizo, elaborado desde el lugar que la modernidad no reconoce, descalifica y busca instrumentalizar, elaborado desde la diferencia colonial, asume que todo conocimiento está geopolíticamente situado" (2010: 72).

Una de las fuentes de la comunicación decolonial es la comunicología de liberación propuesta por el boliviano Luis Ramiro Beltrán (1976). Esta puede ser considerada entre las fuentes del pensamiento decolonial junto a otras elaboraciones del anticolonialismo latinoamericano de entonces.

El programa de investigación de modernidad/colonialidad expresa, desde mediados de los 90, la renovación que vive el pensamiento crítico latinoamericano. Este movimiento que tensiona los conceptos hasta entonces aplicados para dar cuenta de los procesos sociales de la región, tanto como sus presupuestos, aparte de orientarse a la conformación de un "paradigma otro" que se desmarque de la visión eurocéntrica prevaleciente desde el siglo XIX.

La comunicación, pensada desde la teoría decolonial, fue criticada por las teorías hegemónicas de aquellos años, como la comunicación para el desarrollo -vinculada a la idea moderna de progreso-, impregnada de supuestos foráneos surgidos después de la segunda Guerra Mundial y que, si bien se aggiornó, nunca modificó su matriz colonial (Torrico Villanueva, 2013: 2). La comunicación decolonial, heredera de la propuesta de Beltrán, invita a investigar, entender y gestionar los procesos comunicacionales desde una perspectiva que rompa con la lógica de la modernización trasplantada. Que en consecuencia recomponga los instrumentos del conocimiento y que esté orientada por el acceso, el diálogo y la participación democráticos, inscribiéndose en los lineamientos que el programa modernidad/colonialidad asume entre sus antecedentes.

\section{Comunicación e interculturalidad en el marco decolonial}

La particularidad del caso en estudio, que incluye comunidades originarias y procesos de interacciones y negociaciones para la producción de hábitat, demandó pensar estrategias que permitieran poder comunicarnos fluidamente. La intención de generar dispositivos de participación efectiva tuvo sus primeros obstáculos en aspectos culturales. Evidentemente, a pesar de que como equipo de trabajo estábamos posicionados política e ideológicamente

Question, Vol. 1, N. ${ }^{\circ}$ 63, julio-septiembre 2019. ISSN 1669-6581

Instituto de Investigaciones en Comunicación | Facultad de Periodismo y Comunicación Social | Universidad Nacional de La Plata La Plata | Buenos Aires | Argentina 
desde un lugar de reconocimiento del otro, no teníamos ciertas competencias culturales. Entendemos por competencias culturales a "la habilidad para negociar los significados culturales y de actuar comunicativamente de una forma eficaz de acuerdo a las múltiples identidades de los participantes" (Fernándes Fernández y Fernández-Alameda, 2013: 116). Ante la dificultad que representaba no poder derribar estas barreras culturales, comprendimos que el enfoque de comunicación participativa no era más que un mero simulacro: hacíamos que las personas de la comunidad participen de nuestros talleres, pero el impacto de su participación en la implementación de las cocinas de biomasa no variaba de lo que habíamos diseñado previamente. Al advertir esta situación y luego de una intensa búsqueda de materiales teórico-metodológicos congruentes con nuestro posicionamiento paradigmático, arribamos al enfoque intercultural.

Frente a los intentos, propios del colonialismo, de modificar las culturas dominadas para hacerlas lo más próximas a las dominadoras, se aspira a un intercambio entre culturas a los fines de que la tecnología puede ser co-producida con la mediación de un proceso de resignificación del sistema de funcionamiento del equipo tecnológico.

En este punto, consideramos importante dejar sentado que somos conscientes de que posicionarnos desde una perspectiva intercultural no implica -ni posibilita- invisibilizar, ignorar o desconocer las condiciones de desigualdad de las que parte la comunicación con estas otras culturas.

Así pues, si bien nuestro horizonte epistemológico-político es la interculturalidad crítica entendida como:
una herramienta, como un proceso y proyecto que se construye desde la gente -y como demanda de la subalternidad-, en contraste a la funcional, que se ejerce desde arriba. Apuntala y requiere la transformación de las estructuras, instituciones y relaciones sociales, y la construcción de condiciones de estar, ser, pensar, conocer, aprender, sentir y vivir distintas (Walsh, 2010: 78).

La misma autora reconoce que esta interculturalidad no existe, sino que es algo por construir. Al respecto, el posicionamiento político del equipo de investigación/extensión, no es elemento suficiente para garantizar condiciones sociales, relacionales, institucionales, etc. que permitan que la experiencia adopte tal enfoque.

Continuando con la categorización que realiza Walsh (2010) respecto al concepto de interculturalidad, tampoco podemos reconocer en la investigación elementos de la interculturalidad que denomina funcional, la cual "se enraíza en el reconocimiento de la diversidad y diferencia culturales, con metas a la inclusión de la misma al interior de la estructura social establecida" (2010: 77). Sobre esto, consideramos que una interculturalidad que es funcional al sistema dominante (eurocentrista, universalista, moderno, colonial, etcétera), no es interculturalidad más que en su denominación.

Las características de la experiencia responden más a la interculturalidad denominada relacional "que hace referencia de forma más básica y general al contacto e intercambio entre culturas, es decir, entre personas, prácticas, saberes, valores y tradiciones culturales distintas, los que podrían darse en condiciones de igualdad o desigualdad" (Walsh, 2010: 77). Así, podría interpretarse como sinónimos de otros conceptos que suelen asociársele, como multiculturalismo o transculturalismo. Al respecto, María Laura Diez (2004) sostiene que "frente a la multiculturalidad, como categoría 'descriptiva', surge la interculturalidad, como categoría

Question, Vol. 1, N. ${ }^{\circ}$ 63, julio-septiembre 2019. ISSN 1669-6581

Instituto de Investigaciones en Comunicación | Facultad de Periodismo y Comunicación Social | Universidad Nacional de La Plata La Plata | Buenos Aires | Argentina

Página 7 de 10 
'propositiva'” (p. 194), aclarando -además- que no sólo se trata de esta distinción entre lo "descriptivo" y lo "propositivo", sino de la forma en que se describe desde la perspectiva multicultural, como si se tratara de colectivos separados.

Trabajar en la producción social de hábitat desde un enfoque de comunicación e interculturalidad implica un ejercicio complejo de reconocimiento de los sentidos producidos por cada uno de los agentes que participa del proceso estudiado -incluidos los investigadores-, revisar los sentidos coproducidos, entender las disputas de aquello que produce tensiones y reconocer los emergentes.

Aquí y particularmente como elemento para entender los procesos de producción social de hábitat, podemos sumar la noción de comunidades de vida "que presupone la existencia de un grado mínimo de sentido compartido (...) la mayoría de las comunidades de vida, a través de distintas sociedades y épocas, anhelan alcanzar un grado de sentido compartido (...)" (Berger y Luckmann, 1995: 82). Sobre esto, y continuando con la apuesta paradigmática decolonial, podemos afirmar que todos los agentes involucrados en el caso estudiado son herederos de esos otros que el proyecto moderno identificó como bárbaros, incivilizados, arcaicos y subdesarrollados.

Notas

(1) Proyecto de Compromiso Social Universitario (Convocatoria 2016), financiado por la Secretaria de Políticas Universitarias. Oficina de trabajo: Instituto de Investigación en Energía No Convencional (INENCO). Integrantes: investigadores, docentes y estudiantes de grado y posgrado de las Facultades de Humanidades, Ciencias Naturales y Ciencias Exactas de la Universidad Nacional de Salta.

(2) Estamos ante cosmovisiones no occidentales que demandan lo que Boaventura de Sousa Santos (2010) denomina traducción intercultural y que entiende como el objetivo de "establecer una nueva relación de equilibrio dinámico entre el principio de igualdad y el principio de reconocimiento de la diferencia" (p. 8). Santos advierte que la potencial de la traducción no debe car en una versión suave del colonialismo y que la comprensión del mundo es mucho más amplia que la del mundo occidental.

(3) Cuando decimos transferir, nos referimos a la transferencia tecnológica como el movimiento de productos y procesos tecnológicos (o información acerca de ellos) de la universidad y otros centros de investigación a otros actores de la sociedad. Desde esta visión puede definirse como un proceso lineal, unidireccional, de conocimientos y capacidades, mediante el cual la tecnología fluye desde la oferta a la demanda. Además, esta noción de transferencia presume la identidad permanente de un artefacto independientemente del escenario sociohistórico en el que se lo inserta (Escalante, Belmote y Gea, 2013). 


\section{Bibliografía}

Berger, P. y Luckmann, T. (1995). Modernidad, pluralismo y crisis de sentido. Barceona: Paidós Ibérica.

Castro-Gómez, S. y Grosfoguel, R. (2007). El grio decolonial. Reflexiones para una diversidad epistémica más allá del capitalismo global. Bogotá: IESCO-Pensar-Siglo del Hombre Editorial.

Ceballos Guerra, J. C. (2015). Para la Intervención en el hábitat la comunicación: acción social. BDIGITAL, pp. 107-116. Recuperado de http://bdigital.unal.edu.co/2233/1/icc03For19.PDF

Cejas, N. (2013). Procesos Comunicacionales en Prácticas de Co- Construcción del Conocimiento. Córdoba: CEA-UNC.

Cimadevilla, G. (2007). Estado del arte. Trayectos y grises de las teorías y de las prácticas de comunicación y desarrollo. Seminario Internacional Comunicación y Desarrollo, Ediciones INTA, Buenos Aires. Recuperado de http://anterior.inta.gov.ar/activ/comunica/comydes/cimadevilla.pdf

De Sousa Santos, B. (2010). Descolonizar el saber, reinventar el poder. Montevideo: Ediciones Trilce

Diez, M. L. (2004). Reflexiones en torno a la interculturalidad. Cuadernos de Antropología social, 19, 191-213.

Enet, M. (2008). Herramientas para pensar y crear en colectivo, programas intersectoriales de hábitat. Meximo: CYTED-HABYTED-RED XIV.

Escalante, K. N.; Belmonte, S. y Gea, M. (2013). Determining factors in process of sociotechnical adequacy of renewable energy in Andean Communities of Salta, Argentina. Renewable and Sustainable Energy Reviews, 22, pp. 275-288.

Escobar, A. (2003). Mundos y conocimientos de otro modo. El programa de investigación de modernidad/colonialidad latinoamericano. Tábula Rasa, 51-86.

Fernandes Fernádez, M. y Fernández-Alameda, C. (2013). Hacia un nuevo modelo mundial de comunicación intercultural. Methaodos. Revista de Ciencias Sociales, 114-122.

Fuentes Navarro, R. (1998). Perspectivas socioculturales postdisciplinarias en la investigación de la comunicación. En Fuentes Navarro, R. La Emergencia de un campo académico: continuidad utópica y estructuración científica de la investigación en comunicación en Mexico (p. 267). Guadalajara: ITESO.

Galindo, J. (2003). Notas para una comunicología posible. Elementos para una matriz y un programa de configuración conceptual-teórica. Escribanía, 13. Recuperado de http://revistasum.umanizales.edu.co/ojs/index.php/escribania/article/view/3011

Guba, E. y Lincoln, Y. (2002). Paradigmas en competencia en la investigación cualitativa. En Denman, C. y Haro, J. Por los rincones. Antología de métodos cualitativos en la investigación social (pp. 113-145). Sonora: Colegio de Sonora.

Leon Duarte, G. (2006). Sobre la institucionalización del campo académico de la Comunicación en América Latina. Una aproximación a las características estructurales de la investigación latinoamericana en comunicación. Barcelona: Universidad Autónoma de Barcelona.

Mignolo, W. (2003). Un paradigma otro: colonialidad global, pensamiento fronterizo y cosmopolitismo crítico. En Mignolo, W. Historias locales/diseños globales. Colonialidad, conocimientos subalternos y pensamiento fornterizo (pp. 19-60). Madrid: AKAL. 
Moyano, R. (2016). Procesos de estructuración de las prácticas teórico-metodológicas de la investigacion académica de América Latina. ALAICS y FELAFACS (200-2010) (Tesis de doctorado). Facultad de Periodismo y Comunicación Social, Universidad Nacional de La Plata.

Tamayo Sánchez, M. (1997). El análisis de las Políticas Públicas. En Bañon, R. y Carrillo, E. La nueva administración pública. Madrid: Alianza Universidad.

Torrico Villanueva, E. (2004). Abordajes y períodos de la Teoría de la Comunicación. Bogotá: Grupo Editorial Norma.

Torrico Villanueva, E. (2010). La Comunicología de la Liberación, otra fuente para el pensamiento decolonial. Una aproximación a las ideas de Luis Ramiro Beltrán. Quórum Académico, 7(1), 65-77.

Torrico Villanueva, E. (abril de 2013). Una comunicación para salir del desarrollo. IV Congreso Venezolano de Investigadores de la Comunicación. Barquisimeto, Venezuela.

Uranga, W. (15 de Marzo de 2017). La comunicación es acción. Comunicar desde y en las prácicas sociales. Recuperado de https://bit.ly/2SIMSjS

Vanoli, F.; Martínez, V. y Cejas, N. (2018). Procesos comunicacionales en la producción de hábitat: tres inflexiones para un abordaje decolonial. Question, 1(58). https://doi.org/10.24215/16696581e042

Walsh, C. (2010). Interculturalidad crítica y educación intercultural. En Viaña, J.; Tapia, L. y Walsh, C. Construyendo interculturalidad crítica. La Paz-Bolivia: Instituto Internacional de Integración del Convenio Andrés Bello. 